\title{
Subjetivación y Dispositivo: una propuesta de análisis para sujetos en condiciones de pobreza.
}

\section{Subjectification and Dispositif: an analysis strategy for populations in condition of social exclusion}

Francisco Ghisiglieri*

\begin{abstract}
RESUMEN
Con este artículo se aspira a generar una caja de herramientas que permita abordar los procesos de subjetivación en condiciones de pobreza. Para ello, se trabajan las nociones de procesos de subjetivación, dispositivo y pobreza, debido a sus capacidades de incluir en el análisis del sujeto las dimensiones ligadas al poder y al entramado discursivo-social. Se definen como virtudes de este enfoque: analizar las prácticas en su positividad; definir sistemas conservando su carácter acontecimental y singular; aproximarse a un abordaje de la subjetivación en su multiplicidad; y por último, aportar en la búsqueda de mayores dominios de libertad sobre nuestra vida y sus condiciones.
\end{abstract}

Palabras clave: Procesos de subjetivación; dispositivo; dispositivo de desigualación social; pobreza.

\begin{abstract}
This paper aims to build a toolbox to address subjectivation processes in context of social exclusion. For this, the notions of processes of subjectivation, dispositif and poverty are worked on, due to its ability to include in the subject's analysis the dimensions of power and social discourse.There are defined as virtues of this approach: to analyze the practices in its positivity; to define systems preserving its eventual and singular character; to propose an approach to the subjectivity preserving its multiplicity; and, finally, to contribute in the search for greater freedom domains on our life and its conditions.
\end{abstract}

Key Words: Processes of subjectivation; dispositif; social des-equalization dispositif; poverty.

\footnotetext{
* CONICET/Universidad Católica de Córdoba. Doctorando. Argentina. Correo electrónico: francisco.ghisiglieri@gmail.com

Este trabajo se realiza en el marco del proyecto de Tesis Doctoral titulada "Procesos de subjetivación en niños, niñas y adolescentes en contextos de exclusión social" con Beca Cofinanciada UCC-CONICET, dirigido por la Dra. Griselda Cardozo (04/2014 - 04/2019); inscripto en el Doctorado de Psicología de la Universidad Nacional de Córdoba, dirigido por la Dra. Cardozo y la co-dirección de la Dra. Andrea Gigena.

Recepción: 10/08/2019 Aceptación: 20/03/2020 Doi: 10.15517/WL.V15I1.41141
} 


\section{Introducción}

Las condiciones en las que se dan las vidas de los sujetos marginados y marginadas de las grandes ciudades americanas afectan de manera perjudicial su bienestar y capacidades de autodeterminación ${ }^{1}$. Estas condiciones, que sin duda tienen un aspecto material relevante, se engranan en toda una red de condiciones sociales, relacionales, que afectan -también- las subjetividades. Marginación, exclusión, estigmatización, invisibilización hacen parte de un proceso de empobrecimiento generalizado que produjo el ingreso del neo-liberalismo, y que desde entonces, no cesa ${ }^{2}$.

Sin embargo, podemos afirmar que la Psicología muestra una tendencia a enfatizar las dimensiones individuales de los problemas, desconociendo muchas veces las condiciones sociales en las que esos fenómenos acontecen. Esta "tendencia" la transciende, y se relaciona con el modo en que sus conocimientos se articulan con redes de saber-poder. De este modo, podemos ver en la historia de la disciplina, por ejemplo, que a la vez que se instituye como hito fundante el laboratorio experimental de Wundt en Alemania, se desestiman sus trabajos sobre la psicología de los pueblos. En la misma línea, la recepción de la obra freudiana es atrapada por una diferenciación que en ningún momento fue propuesta por el autor. Aquellas producciones que presentaron un interés claro por lo social se definieron como "escritos sociales" y pasaron a ocupar un lugar diferenciado del resto de la producción psicoanalítica.

La Psicología a la que busca inscribirse este trabajo está por fuera de esta tendencia. En América Latina, existen múltiples esfuerzos por realizar la articulación entre lo individual y lo social. De acuerdo con González (2004), la desigualdad imperante en estos territorios es la promotora de la formación de una psicología social que se ha caracterizado por problematizar críticamente las dificultades propias de un campo en el cual lo colectivo y lo singular se enlazan. Pichón Rivière es considerado quien abre el

1 Por caso, véase la situación de tres ciudades Argentinas en Núñez y Ciuffolini (2011), de Chicago en Wacquant (2007), y del panorama general de las grandes ciudades de países dependientes en Davis (2004).

2 En el año 1974 se puede apreciar en Argentina un quiebre que coincide con el ingreso del régimen político-económico neoliberal. Si hasta entonces la pobreza (entendida como carencia de recursos) era un fenómeno presente pero acotado, desde allí se da un proceso de empobrecimiento generalizado de la población que crece hasta el 2003, momento de alguna recuperación para ciertos sectores, pero que en general se estabilizan las proporciones pre-existentes (Arakaki 2011). 
juego a la psicología social en Argentina. A pesar de que sus fundamentos teóricos distan de lo que voy a desarrollar (en su caso: psicoanálisis kleineano, teoría de los roles de George Mead y marxismo, mientras que aquí se retoman desarrollos foucaultianos), sí encuentro una continuidad en que sus trabajos abren una pregunta a la que adscribo: ¿cuáles son las condiciones de existencia en las que los procesos psíquicos se dan? (Pichon-Rivière 1972).

En este trabajo me propongo desarrollar algunas herramientas teóricas que permitan abordar las condiciones en la que los procesos psíquicos en contextos de pobreza se configuran; abonar la búsqueda sobre nociones, términos, conceptos que presenten el potencial de liberar los dominios que circulan en los territorios empobrecidos de nuestras latitudes ¿Qué enfoque para este campo que podemos definir como pobreza?

La perspectiva de los procesos de subjetivación (Foucault 1988; 2008, Castro 2011) aporta a estas discusiones la posibilidad de visibilizar cómo se configuran formas de gobierno de los sujetos que adquieren alcances globales. Encontramos, por caso, una continuidad en la forma de gobierno de las personas que se definen como pobres, con una manera de intentar conducir conductas que a lo largo de América tienen texturas próximas (individualización, estigmatización, marginación, etc.). Asimismo, estos intentos se encuentran siempre con resistencias, con luchas que van en sentido contrario. "Donde hay poder, hay resistencias" sentencia Foucault (1988). Así, por ejemplo, frente a un poder que insiste en desarticular y marginar, encontramos luchas por el "derecho a la ciudad" como un intento de recuperar formas pacíficas y dignas de habitar la ciudad (Borja 2011).

En lo que sigue, nos adentraremos en los debates en los cuales se inscriben las nociones de procesos de subjetivación y dispositivo -con qué conceptos discuten, a qué necesidades intentan dar respuesta, qué implicancias tiene tomar estas herramientas teóricas- y avanzar en una articulación con el campo de problemas de la pobreza.

\section{De la teoría del sujeto a los procesos de subjetivación}

En reiteradas ocasiones se ha llamado la atención sobre el modo en que operan en el campo psicológico las metáforas de interioridad-exterioridad, texto-contexto, singularcolectivo y particular-universal (Fernández 1993, 2007, Fernández y de Brasi 1999). En 
la actualidad, y a la luz de los desarrollos post-estructurales, estos planteos son entendidos como colonizados por una lógica de pensamiento binario, por lo que poco pueden hacer con la multiplicidad de lo real (Deleuze y Guattari 2001). En consecuencia, ¿cómo nominar y entender lo que con aquellas categorías se intentaba dar cuenta? Las nociones de subjetividad y de procesos de subjetivación se proponen saldar esta deuda.

Para delimitar los principales tópicos de esta conceptualización y sus implicancias más generales, se sigue a continuación el esquema propuesto por Bonvillani (2013): subjetividad/identidad, subjetividad/mente/cuerpo y sujeto/subjetividad/subjetivación ${ }^{3}$. Una vez justificado de manera muy somera el contexto conceptual y los debates en los que se inscribe, nos adentraremos con mayor profundidad en la noción específica de procesos de subjetivación entendida desde las conceptualizaciones de Foucault.

La categoría de subjetividad discute necesariamente la idea de identidad como aquello idéntico a sí mismo. Cuando se habla de subjetividad o subjetivación no se busca definir una naturaleza profunda de los seres, ni hallar por fin la esencia personal o aquel rincón oscuro que fuese la propiedad última de una singularidad. Desde esta perspectiva, siempre se está pensando en una construcción social y política. La identidad como categoría conserva su potencia explicativa siempre que se la piense bajo estos supuestos. Un ejemplo de una articulación posible dentro de esta discusión que busca sostener la deconstrucción de identidades fijas, a la vez que potenciar la acción política, es la noción de esencialismo estratégico (Mattio 2009, Spivak 2013).

En lo que refiere al tópico subjetividad/cuerpo/mente, sólo recordar que la vieja tradición platónica, y su dualismo cuerpo-mente, han reinado por mucho tiempo estas temáticas; la mente como equivalente a razón, a logos, desde donde las pasiones relegadas al cuerpo deben ser gobernadas por el pensamiento. Debido a la historia de estas afirmaciones, es de esperar que sus arrastres epistemológicos y metodológicos sean difíciles de revertir. Es de destacar que la afectividad, como dimensión de estudio dentro de las ciencias sociales y humanas, presenta una aparición tardía (Bonvillani 2010). El dominio de la subjetividad implica no sólo abandonar el viejo dualismo cuerpo-mente, sino proponer al campo de lo afectivo como una dimensión significativa a analizar (Fernández 1993, Bonvillani 2013).

3 Dentro de esta delimitación general, se selecciona algunos aspectos propuestos en su trabajo y desarrollaran otros nuevos o diferentes. 
Por último: sujeto/subjetividad/subjetivación. Etimológicamente, subjetividad significa la cualidad de ser sujeto, y este significante se relaciona estrechamente con la idea de sujeción. Fue en los debates propios de lo que se ha llamado estructuralismo entendido como un movimiento crítico hacía la “(...) metafísica occidental moderna (...)" (Ibáñez Gracia, 1996: 26)- donde se definió al sujeto como sujeto a determinadas estructuras (lenguaje, cultura, clases sociales).

Sin embargo, bajo las reconsideraciones post-estructuralistas, se da nuevamente un giro: el sujeto vuelve a entrar en escena. Ya no será el sujeto pasivo determinado por las estructuras, sino que es un sujeto instituido a la vez que instituyente. Está condicionado por las estructuras, pero mantiene un papel activo (Ibáñez Gracia 1996). En este sentido, la noción de subjetivación permite visibilizar los procesos de producción de sí que los sujetos realizan de manera conjunta con los mecanismos de gobierno que los sujetan (Bonvillani 2013, Tassin 2012).

La diferencia entre procesos de subjetivación y subjetividad la entiendo del siguiente modo. Mientras que los procesos de subjetivación en términos generales son las relaciones de saber-poder en las que los sujetos devienen tales, la subjetividad es la resultante de aquellos procesos, es la dimensión de lo social hecho carne, cuerpo, psiquismo. Hablar de subjetividad y no de sujeto busca enfatizar esta dimensión de análisis.

De acuerdo a Castro (2011) la idea de procesos de subjetivación implica, en primer lugar, entender al sujeto desde un abordaje histórico, es decir, bajo la pregunta por los modos en que la historia lo ha atrapado (Foucault, 1988) y las posibilidades de autodefinirse que ese amarre ha dado lugar. En segundo lugar, que el sujeto es una forma, un modo de subjetivación posible, y ello implica necesariamente ser crítico hacía los “universales antropológicos" (Foucault 2002). Es decir, asumir que no existe ningún sujeto a priori, sino que su figura se realiza en su devenir.

Desde ese marco más general, es posible distinguir en la noción de subjetivación un sentido amplio y un sentido estricto (Castro 2011). En sentido amplio, Foucault entiende a la subjetivación como el modo en que los sujetos aparecen objetivados en determinadas relaciones de saber y poder; subjetivación y objetivación se desarrollan mutuamente bajo condiciones que posibilitan unas formas de verdad y no otras. Como lo señala el propio Foucault (1988), su trabajo ha consistido en analizar tres modos de objetivación de los sujetos: los modos de investigación que pretenden estatuto de 
ciencia, los modos de objetivación bajo prácticas divisorias y por último los modos en que un ser humano se convierte a sí mismo en sujeto. A su vez, en sentido estricto, Foucault entiende por modos de subjetivación los procesos en los cuales un sujeto se constituye en sujeto moral; son formas de actividad sobre sí mismos que responden a la capacidad que tiene un sujeto de auto-afectarse.

El enfoque de los procesos de subjetivación es -en definitiva- un modo de abordar las problemáticas relativas al ser humano en cultura con una herramienta que se pretende lo suficientemente amplia como para cobijar la triple dimensión especificada por los desarrollos foucaultianos: saber-poder-ética (Foucault 1988, 2008). Es aquí que la noción de dispositivo se articula, ya que posibilita cartografiar los regímenes de saber-poder en los cuales los procesos de subjetivación acontecen.

\section{Del contexto social a las relaciones de saber-poder}

\subsection{Dispositivo}

Como se ha definido, un concepto eje de este enfoque es el de dispositivo. En el "campo psi"4 es un concepto que se engarzó en las discusiones existentes sobre qué lugar atribuirle y cómo comprender el contexto social en el cual los sujetos despliegan sus acciones. La noción de dispositivo, dentro de una perspectiva foucaultiana, es una propuesta conceptual que acompaña a la de procesos de subjetivación.

Desde un punto de vista histórico, Agamben (2011) sostiene que el antecedente del concepto de dispositivo utilizado por Foucault es la noción de positividad de Hyppolite. Hyppolite la proponía como la llave hegeliana para problematizar la relación entre la historia y la vida de los seres humanos. Ahora bien, en este caso la intención era demostrar cómo se relacionaba la religión histórica, positiva, y la religión natural. En cambio, Foucault se propuso investigar los modos concretos por los cuales los dispositivos actuaban al interior de las relaciones, en los mecanismos y en los juegos del poder (Agamben 2011).

\footnotetext{
${ }^{4}$ Expresión utilizada para denominar las diversas escuelas psicológicas, incluyendo la psicoanalítica.
} 
Por su parte, no existe dentro de la obra de Foucault una definición sistemática del término. Lo más detallado que se encuentra es la aproximación realizada en una entrevista en 1977 (Foucault 1984). Allí lo define como la red de relaciones, la naturaleza del vínculo de un conjunto heterogéneo de elementos (discursivos y no discursivos) que tiene una posición estratégica, una función: responder a una urgencia específica en un momento histórico dado. En la génesis de un dispositivo se diferencian dos momentos: uno en el que prevalece un objetivo estratégico y otro en el que ya el dispositivo se instituye como tal y da lugar a un doble proceso. Por un lado un proceso de sobre-determinación funcional, que hace referencia a un constante juego de reajuste y reacomodación; por el otro, al perpetuo relleno estratégico, es decir, los procesos, los devenires, los efectos que se dan con la puesta en marcha de un dispositivo y que no estaban previstos por ningún sujeto meta o transhistórico (Foucault 1984).

\section{2. ¿Dispositivo de desigualación social?}

Más próximos en el tiempo y el espacio, un antecedente que articula los desarrollos foucaultianos sobre los dispositivos y el análisis de poblaciones marginadas es el trabajo de Ana María Fernández (2011). Propone la idea de dispositivos de desigualación social como aquellos que producen diferencias desigualadas, es decir, dispositivos de poder que en su accionar construyen diferencias asimétricas. Un a priori que posibilita esta acción es la concepción de lo diferente como una anomalía de la identidad. Ello permite una particular relación entre discriminación, desigualdad y explotación bajo un consenso invisibilizado y naturalizado sobre las formas de reproducción de las condiciones actuales, signadas por la apropiación de las potencias y bienes.

Sin embargo, considero que resulta difícil postular a la desigualación como dispositivo. Las relaciones de poder, desde un enfoque foucaultiano (Foucault 2008), tienen como condición de posibilidad a la desigualdad de los elementos que la constituyen. La desigualdad, más que un efecto es de algún modo inherente a las relaciones de poder. Desde aquí, el dispositivo no produce desigualación, sino que la desigualdad de los elementos que lo conforman son un supuesto teórico.

Se podrá sostener, en todo caso, que en realidad el dispositivo opera desde/con la desigualación, la vuelve un elemento estratégico de su proceder. El dispositivo de desigualación produce sujetos vulnerables, por lo que se podría hablar de un dispositivo 
de vulnerabilización. Con todo, la vulnerabilización aparece más como una tecnología de gobierno que el producto de las relaciones de poder.

Un segundo problema que le encuentro a esta postulación es que es un concepto demasiado amplio, capaz de englobar bajo la misma lógica situaciones muy disímiles que pueden ir desde las relaciones de género hasta las de clases económicas. Por ello, en un intento de analizar en cada caso los modos específicos en los que las relaciones de saber-poder se juegan, la pregunta que se plantea es ¿cuál es el tópico que este dispositivo define/produce?

\section{3. ¿Dispositivo Pobreza?}

Entonces ¿No convendría hablar de un dispositivo pobreza? Una de las virtudes de plantearlo de este modo es que permite delimitar con mayor especificidad el campo al que se refiere y pone en primer plano el objeto del dispositivo, es decir, la producción de pobreza. Este concepto visibiliza el gobierno de sujetos definidos como "pobres" a la vez que discute la idea de una ausencia de gobierno o repliegue del Estado (Grinberg 2006, Giavedoni 2013).

Diversos enfoques -como puede ser el estudio realizado en Córdoba con niños, niñas y jóvenes, coordinado por Duschatzky y Corea (2002)- tienen como punto de partida la afirmación de una retirada o disfunción de las instituciones -especialmente del Estadocomo característica primordial de los sectores empobrecidos. Sin embargo, el Estado está presente, y en articulación con otros actores sociales adquiere gran importancia en el gobierno de la vida de los sectores populares.

Como señala Giavedoni (2013), no basta con denunciar los discursos que pregonan la ausencia del Estado con relación al aumento de la pobreza, sino que es necesario captar las nuevas relaciones de poder que sí se están estableciendo. La noción de dispositivo pobreza representa una llave para avanzar en esta labor. A continuación, se definirán algunas de sus características.

La superficie de emergencia de este dispositivo se corresponde con el ingreso de la racionalidad neoliberal y el consecuente fin de la sociedad salarial. La pobreza -realidad que los Estados de Bienestar intentaron ocultar a nivel discursivo- entra de nuevo en escena con la racionalidad neoliberal (Foucault 2007). Esta última, en su resignificación del mundo social, concibe que una tasa de desempleo es "natural" o necesaria, y 
abandona, por lo tanto, la idea de pleno empleo, eje del abordaje de la cuestión social hasta entonces.

Como señala Murillo (2011) $)^{5}$, la pobreza se naturaliza: "pobres hubo siempre”. La pobreza es desde este discurso un fenómeno multicausal, cuyas determinantes pueden ser de diferentes índoles (sociales, políticas) y todas tienen el mismo valor o importancia. Bajo este sencillo y contemporáneo enunciado, se desliga a la pobreza de su relación con la economía -local y sobre todo global- y con el trabajo. Pobres son quienes debido a múltiples circunstancias, a condicionamientos objetivos o subjetivos, se cayeron del juego económico, abandonaron la competencia (Murillo 2011).

Este dispositivo estabiliza bajo el significante "pobres" una multiplicidad de nombres posibles para aquellas personas que presentan desventajas socio-económicas. Capta lo que, por ejemplo, Romero (1997) definía como sectores populares recuperando dentro de la tradición marxista una categoría capaz de aunar dimensiones culturales y materiales- y los significa de este modo.

El incremento de la desigualdad de este período redundó en un nuevo modo de gobierno para una población de trabajos precarizados o directamente desempleada. Para ella, lo que en el Estado de Bienestar eran intentos de protecciones universales que debía garantizar el Estado, bajo la racionalidad neoliberal se tradujeron en subsidios, asistencias focalizadas, centros de salud y educativos empobrecidos, gestión local del riesgo (Murillo 2008, Giavedoni 2013, Grinberg 2015).

Se percibe así cómo se van configurando nuevas condiciones de subjetivación con el ingreso del neoliberalismo. Un régimen de prácticas que toma a su cargo modos de nombrar, de comprender, de actuar que generan efectos específicos en las subjetividades que en ellas se entretejen. Provocan ciertas conductas, ciertas acciones, y dificultan otras. Por ejemplo, es muy diferente comprender a la pobreza como una distribución injusta de recursos la cual se asienta sobre un despojo originario de las posibilidades de producir; que entenderla como un problema que tiene causas múltiples y que por estos problemas se han caído del juego. De igual modo, si pobres hubo siempre, lo sociohistórico se invisibiliza en una definición que no sólo aplasta, solidifica, produce certeza, sino que tiene una consecuencia siniestra: si pobres hubo siempre, el programa ya no será el cambio social, sino la gestión de esa pobreza, paliar sus síntomas.

5 Los análisis de Murillo tuvieron como insumo principal los archivos del Banco Mundial. Esto acentúa la perspectiva desde la que emerge, es decir, la de la gubernamentalidad. 


\section{Reflexiones finales}

Dentro del amplio campo de estudios referidos a procesos de subjetivación, este trabajo sistematiza herramientas analíticas que permiten sostener el análisis desde la triada saber-poder-ética. De esta forma se busca no recurrir a reducciones dentro de la complejidad propia del objeto de estudio. Es decir, sostener la tensión entre las problemáticas referidas a los regímenes de verdad y la gubernamentalidad -sin invisibilizar las prácticas de sí- y la dimensión ética -sin descuidar el estudio de las dimensiones ligadas a los dispositivos de saber-poder-.

Una característica relevante del enfoque propuesto a lo largo de este trabajo es que no postula la existencia de ninguna entidad trascendente, ni oculta. Su dimensión de análisis es la positividad misma de las prácticas, ya sean discursivas o no discursivas. Se mantiene en el plano de su existencia, en las condiciones de posibilidad para que algo pueda ser pensado, actuado o sentido, pero que no se encuentran en ningún otro lado que en las prácticas mismas. Si bien es una dimensión presente, que está allí actuando, sostiene Foucault que esta indagación requiere “(...) cierta conversión de la mirada y de la actitud para poder reconocerla y considerarla en sí mismo" (Foucault, 2002: 145).

En este sentido, permite pensar órdenes, organizaciones, sistemas, estructuras, sin tener que recurrir a concepciones estructuralistas; o con mayor exactitud: define campos de regularidades singulares sosteniendo su carácter acontecimental (Foucault 2002), y define estrategias de poder, sin estrategas (Foucault 2008). Define dispositivos, es decir, conjuntos heterogéneos, red de relaciones, madeja multilineal con cierta orientación, cierta sistematicidad conformada por un ensamble particular de prácticas discursivas y relaciones de poder, pero que responden siempre a determinadas condiciones sociohistóricas, a urgencias específicas; conjuntos que una vez echados a andar, fluctúan en función de procesos de sobredeterminación funcional y rellenos estratégicos a los que su devenir los va instando.

Cabe recordar que dispositivo es una rejilla de inteligibilidad, y en tanto tal, es a definir mediante investigación. Si bien, en sentido amplio, es un dispositivo la escuela, la prisión, etc.; en sentido estricto, lo es sólo a condición de que se pueda especificar sus 
reglas de juego, sus prácticas discursivas propias, sus bordes, su posición estratégica. De este modo, si la pobreza podría constituir un dispositivo es una pregunta abierta.

Por último, este enfoque también presenta una apuesta política, es decir, una reflexión ligada al para qué investigar ¿Para qué conocer la vida de la gente? ¿Para qué estudiar la pobreza? ¿Para gobernarla mejor? ¿Para saciar anhelos epistémicos hacia lo otro? ¿Para obtener cierto estatuto? ¿Quién podría adelantarse a aseverar que nada de esto está en juego de alguna forma? Aún así, el objetivo desde aquí es construir herramientas para ser más libres, conocer para lograr un mayor dominio sobre nuestra vida y sus circunstancias, saber para poder.

\section{Referencias}

Agamben, G. ¿Qué es un dispositivo? Sociológica, 2673 (2011): 249-264.

Arakaki, A. La pobreza en Argentina 1974-2006. Construcción y análisis de información. Buenos Aires:CEPED, 2011.

Bonvillani, A. Jóvenes cordobeses: una cartografía de su emocionalidad política. Nómadas, 32 (2010): 27-43.

Bonvillani, A. Cuerpos en marcha: emocionalidad política en las formas festivas de protesta juvenil. Nómadas, 39 (2013): 91-103.

Borja, J. Espacio público y derecho a la ciudad. Crisis urbana y derecho a la ciudad. Viento Sur, 116 (2011): 36-48.

Castro, E. Diccionario Foucault. Temas, conceptos y autores. Buenos Aires: Siglo XXI, 2011.

Davis, M. Planeta de Ciudades-Miseria: Involución urbana y proletariado informal. New Left Review 126 (2004): 5-34.

Deleuze, G., y Guattari, F. Rizoma. Introducción. (Tercera ed.). México D. F: Ediciones Coyoacán, 2001.

Duschatzky, S., y Corea, C. Chicos en banda. Los caminos de la subjetividad en el declive de las instituciones. Buenos Aires: Paidós, 2002.

Fernández, A. M. La mujer de la ilusión: Pactos y contratos entre hombres y mujeres. Buenos Aires: Paidós, 1993.

Fernández, A. M. Lógicas colectivas de la multiplicidad: Cuerpos, pasiones y políticas. Buenos Aires: Biblos, 2007. 
Fernández, A. M. Hacia los Estudios Transdisciplinarios de la Subjetividad (Reformulaciones académico-políticas de la diferencia). Revista de Investigaciones en Psicología (Instituto de investigaciones de la Facultad de Psicología, Universidad de Buenos Aires), 16 1, (2011): 61-82.

Fernández, A. M., y de Brasi, J. C. Instituciones estalladas. Buenos Aires: Eudeba, 1999.

Foucault, M. El juego de Michel Foucault. En Saber y verdad (pp. 127-162). Madrid: La Piqueta, 1984.

Foucault, M. (1988). El sujeto y el poder. Revista Mexicana de Sociología, 503 (1998): 3-20.

Foucault, M. La arqueología del saber. Buenos Aires: Siglo Veintiuno Editores, 2002.

Foucault, M. Nacimiento de la biopolítica: Curso en el Cóllege de France: 1978-1979 (1a ed.). Buenos Aires: Fondo de Cultura Económica, 2007.

Foucault, M. Historia de la sexualidad I: La voluntad del saber. Buenos Aires: Siglo XXI, 2008.

Giavedoni, J. Foucault, la casa verde y el dispositivo de la pobreza. El gobierno de la nueva cuestión social en América Latina. Kairos. Revista de temas sociales 17 (2013). Recuperado de www.revistakairos.org/k32archivos/FoucaultCasaVerde.pdf.

González Rey, F. La Crítica en la Psicología Social Latinoamericana y su Impacto en los Diferentes Campos de la Psicología. Revista Interamericana de Psicología/Interamerican Journal of Psychology, 382 (2004):351-360.

Grinberg, S. Educación y gubernamentalidad en las sociedades de gerenciamiento. Revista Argentina de Sociología, 4 6 (2006): 67-87.

Grinberg, S. (2015). Dispositivos pedagógicos, gubernamentalidad y pobreza urbana en tiempos gerenciales. Un estudio en la cotidianeidad de las escuelas. Propuesta educativa, 143 (2015): 123-130.

Ibañez Gracia, T. Fluctuaciones conceptuales en torno a la Postmodernidad y la Psicología. Caracas: Universidad Central de Venezuela, 1996.

Mattio, E. ¿Esencialismo estratégico? Un examen crítico de sus limitaciones políticas. Revista Electrónica Construyendo Nuestra Interculturalidad, 45 (2009): 1-11. Recuperado de :http://www.interculturalidad.org/numero05/docs/0301Esencialismo_estrategico-Mattio,Eduardo.pdf 
Murillo, S. Naturalización de la pobreza y la desigualdad. Efectos políticos y subjetivos de las estrategias del Banco Mundial. Revista del Centro Cultural de la Cooperación (2007). Recuperado de http://www.centrocultural.coop/revista/articulo/10/

Murillo, S. Colonizar el dolor. La interpelación ideológica del Banco Mundial en América latina. El caso Argentino desde Blumberg a Cromañón. Buenos Aires: CLACSO Libros, 2008.

Murillo, S. Posmodernidad y Neoliberalismo. Buenos Aires: Ediciones Luxemburg, 2011 .

Nuñez, A. y Ciuffolini, A. Política y territorialidad en tres ciudades argentinas. Buenos Aires: El Colectivo, 2011.

Pichón-Rivière, E. Entrevista a E. Pichon-Riviere- J. Lacan. Respuesta de PichonRivière a un cuestionario de Actualidad Psicológica sobre la relación de su pensamiento y el de J. Lacan. Revista Actualidad Psicológica, 12 (1975).

Romero, L. A. Los sectores populares como sujetos históricos. Última década, 7 (1997). Recuperado de http://www.redalyc.org/articulo.oa?id=19500702

Spivak, G. (2013). En otras palabras, en otros mundos: ensayos sobre política cultural. Buenos Aires: Paidós.

Tassin, E. De la subjetivación política. Althusser/Rancière/Foucault/Arendt/Deleuze. Revista De Estudios Sociales, 43 (2012): 36-49. Recuperado de http://res.uniandes.edu.co/view.php/780/view.php

Wacquant, L. Los condenados de la ciudad. Gueto, periferias y Estado. Buenos Aires: Siglo XXI, 2007. 